\title{
Integrated Structures and Materials Design of Mechanical Components
}

\author{
Geng Chen ${ }^{1,2}$, Lele Zhang ${ }^{1,2^{*}}$, Christoph Broechmann ${ }^{3}$ and Chao Chang ${ }^{4}$
}

In recent years, high fidelity numerical models simultaneously reflecting the behaviors of mechanical systems and their underlying materials have gradually become an indispensable part of modern mechanical and production engineering. In different contexts, the attempt to bridge mechanical engineering with materials science and to replace phenomenological models by the physical ones are named either as integrated computational materials engineering (ICME) or integrated structures and materials design (ISMD). In light of such developments, a large number of models were established. These models not only provide deeper insight into physical relationships within the investigated mechanical systems, but also help with reducing the time and cost required for optimizing the design and processing parameters, especially in new manufacturing technologies such as additive manufacturing (AM). The main objective of this special issue is to introducing state-of-the-art research in the field of integrated structures and materials design, focused on both theories and algorithms of the models, as well as their applications in real engineering problems.

As a response to the Call of Papers, over 8 papers were submitted, and after anonymous review and editors' evaluation, eventually 4 of them were accepted and included in this special issue.

The first paper written by Rajei et al. presents a modeling system for simulating the production and service of high-performance sintered gears. The paper demonstrates how models on different scales and considering different physical effects can be combined in accordance

\footnotetext{
*Correspondence: Ilzhang1@bjtu.edu.cn

${ }^{1}$ School of Mechanical, Electronic and Control Engineering, Beijing

Jiaotong University, Beijing 100044, China

Full list of author information is available at the end of the article
}

to ICME's concept and how do they jointly contribute to the optimization of the product. According to the numerical approach elaborated in this paper, first, using models from both discrete and finite element methods (DEM and FEM), thermomechanical simulations were performed to predict macroscopic behaviors of the gear tooth during the filling and sintering process. Next to that, kinetic Monte Carlo simulation was employed to study microstructural evolution and by using its results as inputs effective elastic properties were determined from the micromechanical homogenization. Then these effective properties were further used in the structural FE model for modeling the heat treatment process. Finally, using the density and hardness value obtained from heat treatment simulation, the load bearing capacity of the gear was calculated employing FE solver with an in-house developed fatigue model. Overall, the paper shows that the ICME approach can be used as a platform for digitalizing the process design and realizing an agile production concept.

Mechanical components composed of metallic or composite materials with metal matrix are often subjected to cyclic thermomechanical loading beyond their elastic limits. Depending on the load level, they may either fail by low cycle fatigue or ratcheting, or remain safe if elastic shakedown happens. Because determining the state of elastic shakedown is a cumbersome task using the conventional incremental FE calculation, in numerous studies great efforts were spent on developing efficient numerical schemes to realize direct method-based calculations. Two contributions in this special issue concern with this issue, dedicated to the macro and micro scales and focused on the methodology and the application aspects, respectively. The paper written by Spiliopoulos and Kapogiannis reviewed a powerful general-purpose 
direct method called RSDM (residual stress decomposition method) which is based on the idea of decomposing the residual stress field by Fourier series. As an extension to this method, the authors introduced in their work a numerical approach they developed for predicting the shakedown limit, the so-called RSDM-S. According to RSDM-S, the shakedown limit can be calculated from a converged series of load factors which starts from a high initial value, and then keeps shrinking until the only remaining term in the Fourier decomposition of the residual stress becomes a constant term. Another paper concerning with shakedown analysis, the one written by Chen et al., deals with the strength prediction of particulate reinforced metal matrix composites (PRMMCs). The study emphasizes the need to view PRMMCs as random heterogeneous materials and to investigate the structureperformance relationship from a statistical perspective. Based on this idea, the paper established an integrated numerical platform consisted of model generation, homogenization, direct method, and statistical analysis to study how endurance limit of the material under tensile and shear stresses are related to the microstructural features. By applying such approach to an exemplary PRMMC material, WC-Co, the study calculated endurance limit of the material and suggested a simplified approach for evaluating the material strength under complicated stress states.

Lightweight structures manufactured by metal AM techniques is another major domain in which the ISMD concepts and models are intensively applied. To study the delamination of sandwich structures with pyramidal lattice truss core, Nuno et al. conducted out-of-plane tensile tests and climbing drum peel tests, and compared test results to both analytical calculations and FEM simulations. Their study confirmed the validity of analytical formula and based on that concluded that the delamination can be avoided by appropriately choosing geometric parameters, especially the strut angle.

Through the papers collected in this Special Issue, we hope the concept of integrated structures and materials design is well demonstrated, and with its potential fully exploited in future the design and engineering of mechanical products can be further improved. The Guest Editor greatly appreciates the authors' excellent contributions to this special issue and the suggestions anonymous reviewers given to the papers.

\section{Acknowledgements}

Not applicable.

\section{Authors' Contributions}

All authors read and approved the final manuscript.
Authors' Information

Geng Chen, born in 1985, is currently a professor at School of Mechanical, Electronic and Control Engineering, Beijing Jiaotong University, China. His research interests include multiscale modeling and structural design based on reliability and fatigue strength.

Lele Zhang, born in 1973, is currently a professor at School of Mechanical, Electronic and Control Engineering, Beijing Jiaotong University, China. Her research interests include passive safety and optimization design.

Christoph Broeckmann, born in 1963, is currently the Head of Institute for Materials Application in Mechanical Engineering, RWTH Aachen University, Germany. His research interests include mechanics of materials and powder metallurgical manufacturing processes.

Chao Chang, born in 1986, is currently an associate professor at School of Applied Science, Taiyuan University of Science and Technology, China. His research interests include micro/nano experiment mechanics and impact dynamics.

\section{Competing Interests}

The authors declare that they have no competing interests.

\section{Author Details}

${ }^{1}$ School of Mechanical, Electronic and Control Engineering, Beijing Jiaotong University, Beijing 100044, China. ${ }^{2}$ National International Science and Technology Cooperation Base on Railway Vehicle Operation of Beijing Jiaotong University, Beijing 100044, China. ${ }^{3}$ Chair and Institute for Materials Applications in Mechanical Engineering, RWTH Aachen University, 52062 Aachen, Germany ${ }^{4}$ School of Applied Science, Taiyuan University of Science and Technology, Taiyuan 030024, China.

Received: 9 November 2021 Accepted: 17 November 2021

Published online: 09 December 2021

\section{Submit your manuscript to a SpringerOpen ${ }^{\circ}$ journal and benefit from:}

- Convenient online submission

- Rigorous peer review

- Open access: articles freely available online

- High visibility within the field

- Retaining the copyright to your article

Submit your next manuscript at springeropen.com 